\title{
Suppression of cathepsin K biomarker in synovial fluid as a free-drug- driven process
}

Journal of Circulating Biomarkers Volume 8: $1-5$ (C) The Author(s) 2019 Article reuse guidelines: sagepub.com/journals-permissions DOI: $10.1177 / 1849454418821819$ journals.sagepub.com/home/cbx (3)SAGE

\author{
Bennett Ma' ${ }^{\prime}$, Gregg Wesolowski², Bin Luo ${ }^{3}$, Traci Lifsted ${ }^{3}$, \\ Keith Wessner', Gary Adamson', Helmut Glantschnig ${ }^{3}$, \\ and Laura S Lubbers ${ }^{3}$
}

\begin{abstract}
Cathepsin K (CatK) inhibitors exhibited chondroprotective and pain-reducing effects in animal models, however, improvements were relatively modest at dose levels achieving maximal suppression of CatK biomarkers in urine. In this report, a previously characterized CatK inhibitor (MK-1256) is utilized to explore the potential of reduced target engagement and/or suboptimal exposure (free drug) as limiting factors to the pharmacological potential of CatK inhibitors in the knee joint. Following oral administration of MK- 1256 at a dose level achieving maximal inhibition of urinary biomarker (helical peptide) in dogs, full suppression of the biomarker in synovial fluid was observed. Subsequent tissue distribution studies conducted in dogs and rabbits revealed that MK-1256 levels in synovial fluid and cartilage were consistent with the free-drug hypothesis. Reasonable projection (within twofold) of drug levels in these tissues can be made based on plasma drug concentration with adjustments for binding factors. These results indicate that the previously observed efficacies in the animal models were not limited by compound distribution or target engagement in the knee tissues.
\end{abstract}

\section{Keywords}

Cathepsin K, MK-1256, osteoarthritis, synovial fluid, cartilage

Date received: 19 April 2018; accepted: I December 2018

\section{Introduction}

Osteoarthritis (OA) is a debilitating disease that is commonly characterized by joint pain and stiffness stemming from progressive cartilage loss, subchondral bone changes, osteophyte formation, and/or inflamed synovium. ${ }^{1,2}$ Excessive degradation of tissue matrices is thought to play an important role in the disruption of the joint homeostasis, as evidenced by elevated proteolytic cleavage of collagen observed in osteoarthritic cartilage along with accelerated turnover of subchondral bone and vertical trabeculae. ${ }^{3}$ Various hydrolytic enzymes catalyzing the breakdown of joint matrices have been implicated for their roles in OA progression, making them appealing targets for the development of disease-modifying anti-OA drugs (DMOADs). ${ }^{1,2}$ Cathepsin $\mathrm{K}$ (CatK), a cysteine protease displaying triple helical collagen hydrolase activity, is capable of disintegrating collagen type I (in bone) and type II (in cartilage) and aggrecan. ${ }^{3,4}$ CatK inhibitors are effective bone antiresorptive agents for the treatment of osteoporosis in clinical trials, demonstrating improvements in bone mineral density and bone strength with robust reductions in collagen

\footnotetext{
'Department of Pharmacokinetics, Pharmacodynamics and Drug Metabolism, Merck \& Co., West Point, PA, USA

${ }^{2}$ Department of Bone Biology, Merck \& Co., West Point, PA, USA

${ }^{3}$ Department of Pharmacology, Merck \& Co., West Point, PA, USA

\section{Corresponding Author:}

Bennett Ma, Department of Pharmacokinetics, Pharmacodynamics and Drug Metabolism, WP75B-200, Merck \& Co., West Point, PA 19486, USA. Email: bennett_ma@merck.com
} 
degradation biomarkers. ${ }^{5,6}$ Early assessments of CatK inhibitors as DMOADs in animal models revealed promising chondroprotective and pain-reducing effects. ${ }^{7-9}$ However, the improvements were relatively modest (approximately 30-50\%) when compared to maximal suppression (approximately $60-80 \%$ ) observed for urinary biomarkers of CatK-mediated collagen degradation. In an attempt to explore the potential of reduced target engagement and/or exposure as limiting factors for the observed efficacy, experiments were conducted to determine biomarker suppression and drug concentration at the knee joint using a previously characterized CatK inhibitor MK-1256. ${ }^{10}$

\section{Materials and methods}

\section{In vivo dog and rabbit studies}

In vivo dog and rabbit studies were conducted in a similar manner as reported previously. ${ }^{10}$ Briefly, adult male beagle dogs received a daily oral dose of MK-1256 (at 7, 70, or $700 \mu \mathrm{g} / \mathrm{kg} ; n=7$ or 8 per dose group) prepared in $0.5 \%$ methylcellulose (MC) with $0.25 \%$ sodium dodecyl sulfate (SDS) or control vehicle. On day 6, serial plasma samples were collected at $0,1,2,4,7$ and $24 \mathrm{~h}$ post-dose with corresponding urine samples also collected in the same 24-h period. After the collection of the last plasma and urine samples on day 7, synovial fluid and articular cartilage samples from both knees were immediately harvested following euthanasia of dogs $(n=4)$ dosed with $700 \mu \mathrm{g} / \mathrm{kg}$ MK-1256 and with control vehicle. Distribution of MK-1256 to synovial fluid and articular cartilage was also studied in 9-monthold ovariectomized female New Zealand White rabbits (approximately $3.5 \mathrm{~kg}$ body weight; $n=5$ ). An oral dose of $30 \mathrm{mg} / \mathrm{kg}$ MK-1256 was administered to the rabbits daily for 4 days, with serial plasma samples collected at $0,1,2,4$, 7 , and $24 \mathrm{~h}$ post-dose on day 4 . Immediately following the collection of 24-h plasma sample, rabbits were euthanized and samples of synovial fluid and articular cartilage from both knees were harvested. All animal studies were conducted in compliance with IACUC policies.

\section{Measurement of biomarker in urine and synovial fluid}

The bone resorption biomarker helical peptide (HP) in dog urine was measured using an enzyme-linked immunosorbent assay followed by normalization to creatinine level determined from the same sample. ${ }^{10}$ For synovial fluid, samples were pretreated with bovine hyaluronidase (Sigma, St Louis, Missouri, USA; 1 unit per $0.2 \mathrm{~mL}$ synovial fluid) at $37^{\circ} \mathrm{C}$ overnight was performed prior to quantification.

\section{Quantitative analysis of MK-I 256 in plasma, synovial fluid, and cartilage}

The concentration of MK-1256 in dog and rabbit plasma was determined by liquid chromatography-tandem mass spectrometry (LC-MS/MS) analysis. ${ }^{10}$ This method was modified for synovial fluid, with samples diluted $1: 10(\mathrm{w} / \mathrm{w})$ with control plasma prior to quantification. For cartilage harvested from knee joint of dog and rabbit, each milligram of tissue was enzymatically digested with $4 \mu \mathrm{L}$ of phosphate-buffered saline containing $2 \mathrm{mg} / \mathrm{mL}$ each of collagenase (Wako Chemicals, Richmond, Virginia, USA) and hyaluronidase at $37^{\circ} \mathrm{C}$ for $24 \mathrm{~h}$ and then processed with a protein precipitation method followed by LC-MS/MS analysis. Binding of MK-1256 to plasma proteins and to cartilage were performed as described by $\mathrm{Ma}$ et $\mathrm{al}^{10}$ and Wang et al, ${ }^{11}$ respectively.

\section{Calculations}

Prediction of drug concentration in synovial fluid and in cartilage was calculated with the following equations

$$
\begin{aligned}
& {[\text { MK-1256 }]_{\text {synovial fluid }}} \\
& \quad=\frac{[\mathrm{MK}-1256]_{\text {unbound, plasma }}}{\text { Fraction unbound of MK-1256 in synovial fluid }}
\end{aligned}
$$

$$
\begin{aligned}
& {[\text { MK-1256 }]_{\text {cartilage }}} \\
& \quad=[\mathrm{MK}-1256]_{\text {unbound, plasma }} \times \text { cartilage binding factor }
\end{aligned}
$$

in which the fraction unbound of MK-1256 in synovial fluid was calculated based on a 2.8 -fold difference in protein content between synovial fluid (approximately $25 \mathrm{mg} / \mathrm{mL}$ ) and plasma (approximately $69 \mathrm{mg} / \mathrm{mL}$ ), ${ }^{11-13}$ and the cartilage binding factor was determined by incubation of MK-1256 with bovine knee cartilage based on the method previously reported. ${ }^{11}$ Statistical analyses, including Student's $t$-test, were performed using the Microsoft Excel software package (Redmond, Washington, USA).

\section{Results}

A dose-dependent decrease in urinary helical peptide (HP) level was observed following oral administration of MK1256 in dogs (Figure 1(a)). This biomarker for collagen degradation was maximally suppressed on day 6 following a daily dose of MK-1256 at $700 \mu \mathrm{g} / \mathrm{kg}$. Full suppression of HP in synovial fluid was observed at this dose level (Figure 1(b)), suggesting that MK-1256 exposure to the knee joint was sufficient to achieve full target engagement. Direct measurement of (total) MK-1256 levels in synovial fluid and cartilage revealed lower drug concentrations in these tissues as compared to that in plasma (Table 1), consistent with the anticipation that knee tissues exhibit less drug binding as a result of reduced protein content. With the apparent applicability of the free-drug hypothesis with regard to MK-1256 distribution in the knee, prediction of drug concentration in synovial fluid was performed with an 


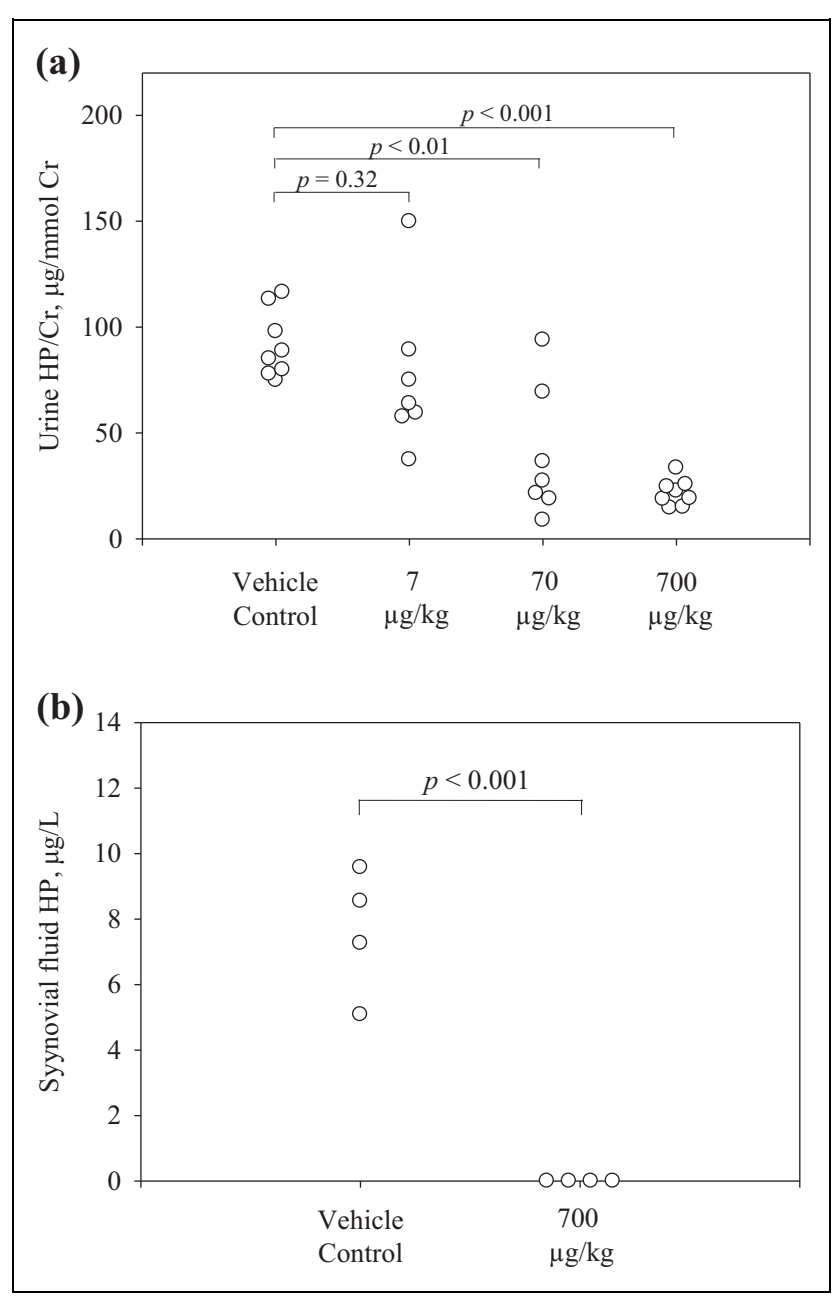

Figure I. Biomarker suppression in dog urine (a) and synovial fluid (b). Male beagle dogs received a daily oral dose of MK- 1256 at 7,70 , or $700 \mu g / \mathrm{kg}(n=7$ or 8$)$. Urine samples were collected on day 6 while synovial fluid samples from both knees were immediately harvested $24 \mathrm{~h}$ after the last dose from the dogs in the high-dose group $(700 \mu \mathrm{g} / \mathrm{kg})$ and control group $(n=4$ per group). Student's $t$-tests were performed and the corresponding $p$ values were reported.

estimated unbound fraction based on the protein content. Projection of MK-1256 concentration in cartilage was obtained by applying a binding factor determined in vitro. Predicted (total) drug concentrations in synovial fluid and cartilage were in reasonable agreement (within twofold) with the measured values (Table 1). In a distribution study conducted in rabbits receiving a daily dose of $30 \mathrm{mg} / \mathrm{kg}$ MK-1256, the prediction drug concentrations in synovial fluid and cartilage were also within twofold of the observed values (Table 1), indicating that the presented approach to predict drug concentrations in the knee tissues is applicable across species.

\section{Discussion}

CatK inhibitors have been suggested to display certain characteristics of DMOADs, exhibiting chondroprotective and pain-reducing effects in various animal models. ${ }^{7-9}$ In those cases, modest improvements were observed at dose levels achieving maximal suppression of urinary biomarkers for collagen degradation. To investigate the potential of reduced target engagement and/or drug level in the knee joint as contributing factors for limited efficacy, experiments were conducted in the current study to measure both the CatK activity biomarker (HP) level and the distribution of a highly selective CatK inhibitor (MK-1256) in the knee. Maximal suppression of urinary biomarker was observed in dogs receiving a daily dose of MK-1256 at $700 \mu \mathrm{g} / \mathrm{kg}$, consistent with the measured plasma drug concentration $\left(C_{\mathrm{avg}}\right.$ : $0.52 \mu \mathrm{M}$; Table 1) being 43-fold higher than the reported half maximal effective concentration $(12 \mathrm{nM}){ }^{10}$ At this dose level, full suppression of HP in synovial fluid was also achieved, suggesting that the unbound drug concentrations in synovial fluid and plasma closely resembled each other - a phenomenon that had been observed for several nonsteroidal antiinflammatory drugs (NSAIDs). ${ }^{14,15}$

Synovium is a highly vascularized membrane that encapsulates synovial fluid present in the articular cavity. ${ }^{14,15}$ The porous nature of the membrane allows small molecules to diffuse in and out of the joint cavity efficiently. Pharmacokinetic studies conducted in human with intra-articular administration of NSAIDs showed that these compounds efficiently diffused out of the synovial fluid, with half-life values observed in synovial fluid similar to those detected in plasma. ${ }^{14,15}$ Application of the non-compartmental analysis revealed that the area under the concentration-time curve (AUC) measured in synovial fluid obtained after oral administration of NSAIDs was in good agreement with the plasma AUC. Consistent with this observation, the average (total) plasma drug concentrations determined in the current study were in reasonable agreement (within twofold) with the drug concentrations in synovial fluid after adjusting for protein binding (Table 1). As the unbound drug concentration in synovial fluid approached equilibrium with the unbound fraction in plasma at steady state, full suppression of the biomarker in synovial fluid was observed at the dose level that achieved maximal inhibition of urinary biomarker (Figure 1). While biomarker and the pharmacodynamics effect of MK-1256 was not directly assessed in articular cartilage, predicted and observed MK-1256 exposure data are congruent with inhibition of CatK in this compartment in dogs and rabbits. Taken together, the presented results in the animal models indicate that CatK biomarker suppression in synovial fluid is a free-drug-driven process.

Early human pharmacokinetics studies conducted with NSAIDs showed evidence of applicability of free-drug hypothesis regarding compound distribution to the knee joint. ${ }^{14}$ For compounds exhibiting extensive binding to plasma proteins (e.g. protein binding of $>98 \%$ ), drug concentration in synovial fluid in reference to that in plasma 
Table I. Observed and predicted MK-I 256 in plasma, synovial fluid and cartilage in dogs and rabbits.

\begin{tabular}{|c|c|c|c|c|c|c|c|c|c|}
\hline $\begin{array}{l}\text { Species } \\
\text { (subjects) }\end{array}$ & $\begin{array}{l}\text { Dose } \\
(\mathrm{mg} / \mathrm{kg})\end{array}$ & $\begin{array}{c}\text { Plasma }^{\mathrm{a}} \\
\text { concentration } \\
(\mu \mathrm{M})\end{array}$ & $\begin{array}{c}\text { Fraction } \\
\text { unbound } \\
\text { in plasma } \\
(\%)\end{array}$ & $\begin{array}{l}\text { Estimated } \\
\text { fraction } \\
\text { unbound } \\
\text { in synovial } \\
\text { fluid (\%) }\end{array}$ & $\begin{array}{c}\text { Predicted } \\
\text { synovial fluid } \\
\text { concentration } \\
(\mu \mathrm{M})\end{array}$ & $\begin{array}{c}\text { Observed } \\
\text { synovial fluid } \\
\text { concentration } \\
(\mu \mathrm{M})\end{array}$ & $\begin{array}{c}\text { Cartilage } \\
\text { binding } \\
\text { factor }\end{array}$ & $\begin{array}{c}\text { Predicted } \\
\text { cartilage } \\
\text { concentration } \\
(\mu \mathrm{M})\end{array}$ & $\begin{array}{c}\text { Observed } \\
\text { cartilage } \\
\text { concentration } \\
(\mu \mathrm{M})\end{array}$ \\
\hline $\operatorname{Dog}(n=4)$ & 0.7 & $0.52 \pm 0.15$ & 30 & 54 & 0.29 & $0.15 \pm 0.07$ & 1.2 & 0.18 & $0.27 \pm 0.11$ \\
\hline Rabbit $(n=5)$ & 30 & $2.1 \pm 0.2$ & 40 & 65 & 1.3 & $0.90 \pm 0.31$ & 1.2 & 1.0 & $1.3 \pm 0.3$ \\
\hline
\end{tabular}

${ }^{\mathrm{a}}$ Reported as mean \pm standard deviation for average concentration within a $24-\mathrm{h}$ period.

should be proportional to the (albumin) protein concentration ratio between these two matrices - an observation that was reported for inhibitors of matrix metalloprotease following oral administration of compound to rabbits. ${ }^{11}$ With the consideration of a 2.8 -fold difference in protein content between synovial fluid and plasma, drug binding to synovial fluid can be estimated from the plasma protein binding value determined in vitro. ${ }^{13}$ This approach allows a projection of drug concentration in synovial fluid based on plasma drug level when equilibrium of unbound drug is reached between these matrices. Reasonable prediction (within twofold) was observed in the current study as well as data reported by Wang et al. ${ }^{11}$ and Day et al. ${ }^{14}$ confirming the feasibility of the current approach to estimate drug level in synovial fluid based on plasma drug concentration and the unbound fraction of drug in plasma.

In conclusion, the current study confirmed that the distribution of MK-1256 to the knee joint is consistent with the free-drug hypothesis, leading to full suppression of local CatK biomarker in the synovial fluid at the dose level achieving maximal inhibition of urinary biomarker. The drug levels in synovial fluid and cartilage can be reasonably estimated based on plasma drug concentration with adjustments for binding factors. These results indicated that the observed efficacies in the animal models of OA were likely not limited by compound distribution or target engagement (i.e. CatK inhibition) in the knee tissues.

\section{Declaration of conflicting interests}

The author(s) declared the following potential conflicts of interest with respect to the research, authorship, and/or publication of this article: All authors are current or former employees of Merck \& Co., who were employed at the time the experiment was conducted. The authors alone are responsible for the writing and content of this article.

\section{Funding}

The author(s) disclosed receipt of the following financial support for the research, authorship, and/or publication of this article: This work was funded by Merck \& Co.

\section{ORCID iD}

Bennett Ma (D) https://orcid.org/0000-0002-8494-9875

\section{References}

1. Karsdal MA, Bay-Jensen AC, Lories RJ, et al. The coupling of bone and cartilage turnover in osteoarthritis: opportunities for bone antiresorptives and anabolics as potential treatments? Ann Rheum Dis 2014; 73: 336-348.

2. Thysen S, Luyten FP, and Lories RJ. Targets, models and challenges in osteoarthritis research. Dis Model Mech 2015; 8: $17-30$.

3. Mort JS, Beaudry F, Théroux K, et al. Early cathepsin K degradation of type II collagen in vitro and in vivo in articular cartilage. Osteoarthr Cartil 2016; 24: 1461-1469.

4. Aguda AH, Panwar P, Du X, et al. Structural basis of collagen fiber degradation by cathepsin K. Proc Natl Acad Sci USA 2014; 111: 17474-17479.

5. Cheung AM, Majumdar S, Brixen K, et al. Effects of odanacatib on the radius and tibia of postmenopausal women: improvements in bone geometry, microarchitecture, and estimated bone strength. J Bone Miner Res 2014; 29: 1786-1794.

6. Eastell R, Nagase S, Small M, et al. Effect of ONO-5334 on bone mineral density and biochemical markers of bone turnover in postmenopausal osteoporosis: 2-year results from the OCEAN study. J Bone Miner Res 2014; 29: 458-466.

7. Hayami T, Zhuo Y, Wesolowski GA, et al. Inhibition of cathepsin $\mathrm{K}$ reduces cartilage degeneration in the anterior cruciate ligament transection rabbit and murine models of osteoarthritis. Bone 2012; 50: 1250-1259.

8. Connor JR, LePage C, Swift BA, et al. Protective effects of a cathepsin K inhibitor, SB-553484, in the canine partial medial meniscectomy model of osteoarthritis. Osteoarthritis Cartilage 2009; 17: 1236-1243.

9. McDougall JJ, Schuelert N, and Bowyer J. Cathepsin K inhibition reduces CTXII levels and joint pain in the guinea pig model of spontaneous osteoarthritis. Osteoarthritis Cartilage 2010; 18: 1355-1357.

10. Ma B, Luo B, Eluer DH, et al. Applicability of in vitro-in vivo translation of cathepsin $\mathrm{K}$ inhibition from animal species to human with the use of free-drug hypothesis. Naunyn-Schmiedeberg Arch Pharmacol 2017; 390: 435-441.

11. Wang $\mathrm{Q}$, Glasson $\mathrm{S}$, Raut $\mathrm{U}$, et al. In vitro-in vivo correlation on delivery of drug candidates to articular cartilage. Pharm Res 2008; 25: 1641-1646. 
12. Chen CPC, Hsu CC, Chen RL, et al. Changes of synovial fluid protein concentrations in supra-patellar bursitis patients after the injection of different molecular weights of hyaluronic acid. Exp Gerontol 2014; 52: 30-35.

13. Kalvass JC and Maurer TS. Influence of nonspecific brain and plasma binding on CNS exposure: implications for rational drug discovery. Biopharm Drug Dispos 2002; 23: 327-338.
14. Day RO, McLachlan AJ, Graham GG, et al. Pharmacokinetics of nonsteriodal anti-inflammatory drugs in synovial fluid. Clin Pharmacokinet 1999; 36: 191-210.

15. Larsen C, Østergaard J, Larsen SW, et al. Intra-articular depot formulation principles: role in the management of postoperative pain and arthritic disorders. J Pharm Sci 2008; 97: 4622-4654. 